\section{Making a Dispersion of Silica Particle for Microscopic Analysis:}

Silica particles don't know that they are supposed to separate and stay away from each other when dispersed in a liquid followed by a droplet of this liquid suspension being placed on a solid surface. They tend to agglomerate very quickly leading to a difficult-to-analyze situation, especially using automated means of analysis. The size range expected could be on the order of 3 to $6 \mathrm{~nm}$.

This is the ideal application for the camphor/naphthalene method which I described several years ago. Credit for the technique, or at least the one who taught it to me was an innovative microscopist then working at the DuPont Experimental Station in Wilmington, DE by the name of Robert $P$. Schatz, in about 1968, now deceased:

Take a $60 \%$ camphor $/ 40 \%$ naphthalene mixture and heat it to 20 or so degrees above room temperature on a hot plate in a small beaker or flask, the two organics are miscible in each other and this is the eutectic composition.

Once a clear liquid, add a small amount of the silica (not more than $0.1 \%$ ), which disperses quite readily. Then, using a pipette, take out some liquid and put a drop onto a carbon coated glass slide, at which time the drop is instantly frozen solid (it is at room temperature). Put the slide into your vacuum evaporator to pump out all night and the "magic" is that the solid eutectic sublimes at room temperature at such a rate that by morning it is completely gone, leaving the silica particles uniformly dispersed on the carbon film!

The rest is obvious. You can pick this up on a grid as is, or in order to bring out more contrast, Pt/C shadow, probably using an angle not more than 30 degrees. You can float the "replica" off of the slide directly onto a grid and viola! You have particles completely dispersed, virtually no doublets or triplets, and a field quite amenable for automated image analysis (as a bonus).

One important further suggestion: Some times these silica particles tend to fuse together as little "chains". If you suspect this is happening, be sure to take the micrographs as stereo pairs because you can in fact capture this three dimensional spatial information.

\section{Charles A. Garber, SPI Supplies}

Disclaimer: We do not sell either the camphor or naphthalene so have no vested interest in whether people use this method or not. It is just a really neat method for the preparation of fine particle samples in this size range. We are, however, obviously set up to use this method as a service for others.

\section{OIL DROPS ON AN EDS DETECTOR:}

A) Diagnosing and Preventing Contamination:

Oil drops on an EDS detector in an instrument with a vacuum system based on an oil diffusion pump could be from either the diffusion pump, or the rotary backing pump, or both. I don't know any easy way to tell which: however, if a few drops of it can be collected, an infra-red spectrum could be run, and that should give a definitive identification.

In the past, we have had trouble with oil collecting on the EDS detector in one of our SEMs and an FTIR spectral analysis showed it to be mainly from the rotary vane backing pump. As discussed on p. 144 of my book, "Vacuum Methods in Electron Microscopy", the molecules of the roughing pump oil get broken down into volatile low molecular weight fragments by the mechanical rubbing of the parts inside the pump, and these fragments readily migrate into the backing line and hence through the diffusion pump and into the vacuum system. Most diffusion pump oils are quite resistant to thermal and oxidative degradation, and so are unlikely to be a major contributor unless the vacuum system on your instrument is poorly designed or your diffusion pump has been subjected to one of the improper operating situations described on p. 214-223 of $V M$ in EM.

Although there is not much that can be done to reduce contamination from the diffusion pump once an instrument is built (except to avoid the operating hazards just mentioned), there are two fairly effective ways of dealing with contamination problems arising from the roughing pump:

1) Give the instrument a good cleaning and then install a good foreline trap, which must then be faithfully maintained to preserve its effectiveness (VM/EM p.147-149).

2) Use an inert gas purging system, which is brought into operation when the instrument can be put into the standby mode, to attempt to sweep the oil molecules back out of the vacuum system - VM/FM p. 145

\section{Wilbur C. Bigelow, University of Michigan Ann Arbor}

B) Cleaning EDS Detector Windows - I:

In any event, the contaminating oil must be removed from the EDS detector window, otherwise it will begin to alter the detector sensitivity. This can be a very tricky process, and should be done with a great deal of care. It is best to check with the manufacturer of your detector for their recommendations before you try anything. However, we have cleaned the standard beryllium detector windows several times in the past by using squeeze bottle or a dropper to run a bit of petroleum ether over the face of the window. You must, of course, to be very careful not to puncture the window, but then the whole operation is one of high risk to begin with. We have even been able to do this without removing the detector from the SEM by using a long dropper and collecting the petroleum ether on a tissue as it runs off the detector.

Petroleum ether is basically a low-boiling hydrocarbobn fraction that is a reasonably good general solvent, especially for the hydrocarbon-based rough pump oils, but which does not attack epoxies and which also evaporates quite readily and cleanly. Do NOT use a solvent such as acetone, methyl-ethyl ketone, or an alcohol, which may attack the epoxy cement normally used to hold the window in place.

Wilbur C. Bigelow, University of Michigan Ann Arbor

\section{C) Cleaning EDS Detector Windows - II}

The above method is fairly similar to ours. Silicone pump oil is little used in EMs, but if it is, and kerosene is used to clean the detector, a second solvent would be needed to remove the kerosene residues.

Reagent grade methanol has been suggested (by an EDS manufacturer) as a suitable solvent for hydrocarbon pump oils. Presumably this was recommended because it did not affect the Be window's glue and it is a good solvent of vacuum oils and fluids. Petroleum ether is likely a better choice.

We too could leave the detector in place, and I collected the used solvent in a Petri dish and tissue.

The significant difference in our methods was the application. Because the window is recessed and our detector had some tilt, it seemed that little solvent would reach the window if the methanol or petroleum ether was just dripped from the top. This is the method we adopted:

Use a polyethylene Pasteur type pipette with a fairly large bulb. Pull the tip into a fine capillary after brief ( $5-10$ seconds) heating in a low flame. (Tip: move and rotate pipette while heating: pull vertically, this keeps the tip straight while the plastic sets). Cut the tip so that about $100 \mathrm{~mm}$ of capillary tip and a small bore, perhaps $0.2 \mathrm{~mm}$, is available.

FILL the bulb with methanol; releasing air once or twice by up-turning and squeezing the bulb. Filling is slow but no trouble.

Slide the stand until the pipette tip is within 20 to $40 \mathrm{~mm}$ of the Continued on Page 22 


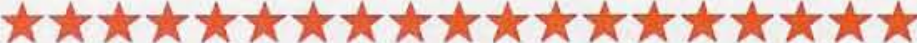

\section{Microscopy 101 - Continued from Preceding Page}

window. Hold pipette with one hand (hand should rest, never work mid-air) near the tip and gently squeeze the bulb. The aim is to 'hit with little force the upper rim of the Be window - where it is supported. Less force on the window and also slightly better coverage is achieved with the methanol stream directed well away from the perpendicular.

Freehand application is possible with care and a steady hand, but most people would be happier mounting the pipette horizontally in a low "retort" stand, at the Be window's level.

A side benefit of well-tilted detectors is that cleaning is much easier: simply use a suitable vial half filled with solvent and slide the detector snout into this. Slightly swirl and cleaning is done.

$$
\text { Jim Darley, ProSciTech (Australia) }
$$

\section{Preventing Curling of Thick Plastic Sections:}

I have been doing plastic sectioning for $20+$ years, mostly glycol methacrylate (GMA), but I started out with methyl methacrylate (MMA). One to seven micrometer plastic sections do not ribbon, unless you put a dab of rubber cement on the top and bottom of the block, but usually we pick them up one section at a time. Curling is very common, so what I do is start the sectioning but do not finish, keep the section attached to the block, then use a brush or fine forceps and unroll it, pulling at a diagonal (leaving it attached lets you pull without completely pulling the section off). When it is mostly open and flat, complete the sectioning stroke, releasing the section.

I used to slide the MMA section onto a spatula, keeping the section wet with ethanol, and then sliding it off the spatula onto a slide on a hot plate. Keep dropping alcohol onto it, and it should flatten out.

GMA is much easier to pick off the block. Do the same thing, but keep everything very dry, pick up the section with a fine forceps and drop it onto a water bath and it will flatten out. Scoop onto a slide from the water.

\section{Patsy Ruegg, University of Colorado Health Sciences Center}

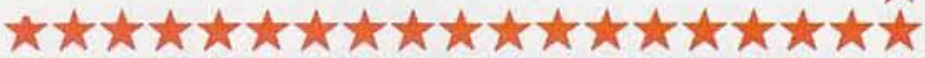

\section{EmpIOYmEnT OpRORTUnITIES}

- Post Doc Positions: High resolution in situ microscopy. Corrosion advanced battery, electrochemistry, polymer, materials science, biologySPM. Several locations: U.S., Japan, Europe.

http://www.molec.com/jobs/postdoc.html

\section{USED EOUIPMENT FOR SALE}

Philips EM-400T-FEG (field emission gun) TEM complete with EDAX ECON detector, EDAX PV 9800 analyzer, STEM (PW6585) unit and air cooled water chiller. Also available: Edax detector (with $\mathrm{Be}$ window) and Edax 9900 analyzer. All items are in good working condition. For details call: (203)389-6065 or FAX (203)387-3574.

\% MILITARY RESEARCH LAB IS CLOSING - Military contractor is selling at drastically reduced prices its Reichart Polycut S motorized sliding microtome, refrigerated and rotary microtomes, Sorvall ultramicotome, Gatan Model 600 dual ion mill. Fisher embedding center, stereo microscopes, Perkin Elmer microdensitometer and LECO sulfur analyzer. For specification sheets, call: (202)544-0836.

\section{CAMERA ADAPTABLE MICROSCOPE}

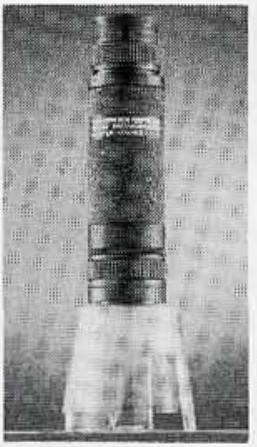

MACROSCOPE 25 provides $25 x$ magnification over an 8 $\mathrm{mm}$ wide field of view. This low cost instrument has fully corrected flat field optics and includes a measureing scale calibrated in both millimeters and inches. The image is positionally correct making it extremely easy to use. Typical applications include field entomology, textiles secimen set for EM, forensic document examinations, horticulture, production quality control in manufacturing.

For further information on this and other MACROSCOPE Portable microscopes contact RF Inter-Science Co. (516)698-4799, Fax: (516)698-4988, eMail: RichF516 @aol.com or visit our webside: http://members.aol.com/ RichF516

\section{Circle Reader Inquiry \#40}

TopoMetrix Introduces Accurex ${ }^{\text {TM }}$ II MS:

\section{First Dedicated AFM for Optical Storage Applications}

TopoMetrix Corporation introduces the first atomic force microscope (AFM) designed specifically for optical storage applications. The Accurex II MS makes three dimensional, high resolution surface measurements of $D V D, C D$, and related stampers and discs.

The Accurex II MS can scan areas from 100 microns by 100 microns, to areas of less than 1 micron on a side, and features up to 10 microns high (or deep). Scan resolution (in all dimensions) is 0.1 nanometer.

Measurement accuracy in all three dimensions is provided by a patented TopoMetrix design that is well proven, incorporated in over 300 TopoMetrix AFM systerns worldwide.

The Accurex II MS comes in two configurations:

The Inspection Configuration is designed to be operated by technicians. Instrument set-up is procedure driven, so that the technician follows instructions provided by the system computer, and selects the analyses to be run. Standard measurements, such as pit lengths and depths, are automated.

The Research Configuration provides the process development scientist with all AFM operating modes, as well as TopoMetrix SPMLab ${ }^{n u}$ software for the greatest flexibility and power in data acquisition, image display and analysis, and screen editing. The Research Configuration also includes the automated analysis capabilities of the Inspection Configuration.

For further information, contact Paul West, VP of Marketing \& Sales, TopoMetrix Corporation: (408)982-9700, Fax: (408)982-9751.

Circle Reader Inquiry \#41

\section{PC-Based Image/X-ray Analyzer Exclusively for SEMs}

AutoSEM 1 is a PC based image analyzer and $x$-ray analyzer for an SEM capable of particle/feature size, shape and $\mathrm{x}$-ray analysis for thousands of features, live/interactively or automatically without operator attendance. Ideal for new SEMs or an upgrade for existing SEMs. With AutoSEM 1 you have an integrated SEM/image/x-ray analytical instrument with greater performance, ease of use and better results. It provides complete image/x-ray analysis reports including statistics in standard spread sheet format; digital color coded $x$-ray maps based on composition rather than single element; digital high resolution imaging, image enhancement, image archiving, and inexpensive photo quality printed images Applications are for air, metal inclusions, water, asbestos, solid particle pollution, GSR, ceramics, and many others

\section{Advanced Research Instruments Corp.}

2434 30th Street, Boulder, CO 80301

Tel.: (303)449-2288, Fax: (303)449-9376, eMail: aricorp@aricorp.com Circle Reader Inquiry \#42 